\title{
Implementing Pediatric Asthma Pathways in Community Hospitals: A National Qualitative Study
}

\author{
Corrie E McDaniel, DO ${ }^{1 *}$, Melanie Jeske, MS², Esther M Sampayo, MD, MPH³, Peony Liu, MD,
} Theresa A Walls, MD, MPH${ }^{5}$, Sunitha V Kaiser, MD, MSc ${ }^{6}$

\begin{abstract}
${ }^{1}$ Department of Pediatrics, University of Washington, Seattle, Washington; ${ }^{2}$ Department of Social and Behavioral Sciences, University of California, San Francisco, California; ${ }^{3}$ Section of Emergency Medicine, Baylor College of Medicine, Houston, Texas; ${ }^{4}$ Kaiser Permanente Southern California Medical Group, San Diego, California; ${ }^{5}$ Department of Pediatrics, Children's Hospital of Philadelphia, Philadelphia, Pennsylvania; ${ }^{6}$ Department of Pediatrics, University of California, San Francisco, San Francisco, California.
\end{abstract}

BACKGROUND: Pathways can improve the quality of care and outcomes for children with asthma; however, we know little about how to successfully implement pathways across diverse hospital settings. Prior studies of pathways have focused on determining clinical effectiveness and the majority were conducted in children's hospitals. These approaches have left crucial gaps in our understanding of how to successfully implement pathways in community hospitals, where most of the children with asthma are treated nationally.

OBJECTIVE: The aim of this study was to identify the key determinants of successful pediatric asthma pathway implementation in community hospitals.

METHODS: We conducted a qualitative study of healthcare providers that served as project leaders in a national collaborative to improve pediatric asthma care. Data were collected by recording semi-structured discussions between project leaders and external facilitators (EF) from December 2017 to April 2018. Using inductive thematic analysis, we identified the themes that describe the key determinants of pathway implementation.

RESULTS: Project leaders $(n=32)$ from 18 hospitals participated in this study. The key determinants of pathway implementation in community hospitals included (1) building an implementation infrastructure (eg, forming a team of local champions, modifying clinical workflows, delivering education/ skills training), (2) engaging and motivating providers (eg, obtaining project buy-in, facilitating multidisciplinary collaboration, handling conflict), (3) addressing organizational and resource limitations (eg, support for electronic medical record integration), and (4) devising implementation solutions with $\mathrm{EFs}$ (eg, potential workflow modifications).

CONCLUSIONS: Our identification of the key determinants of pathway implementation may help guide pediatric quality improvement efforts in community hospitals. EFs may play an important role in successfully implementing pathways in community settings. Journal of Hospital Medicine 2020;15:35-41. () 2020 Society of Hospital Medicine

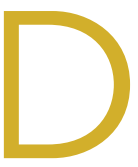

espite the widespread availability of evidence-based guidelines, ${ }^{1}$ there is inappropriate variation in the care and outcomes for children with asthma in both the emergency department (ED) and the inpatient setting. ${ }^{2-6}$ Operational versions of evidence-based guidelines known as "pathways" have been shown to improve adoption of evidence-based guidelines, quality of care, and health outcomes for children with asthma. ${ }^{7-14}$ However, little is known about how to successfully implement pathways outside of free-standing children's hospitals. ${ }^{15-19}$

The majority of children with asthma in the United States are cared for in community hospitals, which provide services for *Corresponding Author: Corrie E. McDaniel, DO; E-mail: corrie.mcdaniel@
seattlechildrens.org; Telephone: 206-818-9821.

Published online first September 18, 2019.

Find Additional Supporting Information in the online version of this article.

Received: May 21, 2019; Revised: July 30, 2019; Accepted: July 31, 2019

๑ 2020 Society of Hospital Medicine DOI 10.12788/jhm.3296 both adults and children. ${ }^{20}$ However, prior studies of pediatric asthma pathways have largely excluded community hospitals. These studies primarily focused on determining clinical effectiveness, rather than detailing the implementation process. These approaches have left critical gaps that hinder our ability to implement pathways and improve care in community hospitals, which have unique barriers and less resources. ${ }^{21,22}$ Therefore, understanding the process of pathway implementation in community hospitals is critical to improving care for children. ${ }^{22}$ Our objective was to identify the key determinants of successful pediatric asthma pathway implementation using a national sample of community hospitals. This knowledge can guide hospital leaders and healthcare providers in efforts to improve pediatric care and outcomes in these settings.

\section{METHODS}

Study Setting, Design, and Population

In Fall 2017, the Value in Inpatient Pediatrics (VIP) network launched PIPA, Pathways to Improving Pediatric Asthma care. ${ }^{23}$ The VIP network, a part of the American Academy of Pediat- 
rics (AAP), aims to improve the value of care delivered to any pediatric patient in a hospital bed, from rural to free-standing children's hospitals. ${ }^{24}$ PIPA used a learning collaborative model ${ }^{25}$ and recruited local project leaders (physicians, nurses, respiratory therapists (RT), and pharmacists) from 89 hospitals around the country. PIPA provided these hospital teams with asthma pathways and several resources for implementation support, including educational meetings, quality improvement (Ql) training, audit and feedback, and facilitation. Facilitation is a process of interactive problem-solving and support that occurs in the context of a supportive interpersonal relationship and a recognized need for improvement. ${ }^{26}$ A facilitator, or a "coach", is an external expert who provides project mentorship and assists the process of making meaningful changes to improve patient care. ${ }^{26}$ Facilitation was provided by external consultants with $\mathrm{Q}$ lexpertise.

For this qualitative study, facilitators conducted semi-structured interviews with a convenience sample of project leaders from community hospitals participating in PIPA, with some interviews including multiple project leaders (eg, nursing, inpatient, and Emergency Department [ED] leaders). Verbal consent was obtained from all participants. No incentives were provided. This study was approved by the AAP institutional review board.

\section{Data Collection}

We used the constructs described in the Consolidated Framework for Implementation Research (CFIR) ${ }^{27}$ and adapted those salient to pediatric asthma pathways to develop an interview guide that was used with all participants (Appendix 1). The CFIR offers an overarching typology to understand what works where and why across five major domains that influence implementation: intervention characteristics, inner setting (hospital), outer setting (economic, political, and social context of the hospital), characteristics of the individuals involved, and the process of implementation. Data were collected across these domains to inform our analysis of the key determinants of pediatric asthma pathway implementation in community hospitals.

Interviews were conducted by phone from December 2017 to April 2018 (first four months of pathway implementation). Interviews lasted 30-60 minutes and were recorded and transcribed verbatim. Transcripts were edited for accuracy using the audio recordings. As data collection occurred concurrently with analysis, the interview guide was iteratively revised to reflect new insights and patterns that emerged from our analysis. All sites were anonymized in the data analysis. New interviews were coded until thematic saturation was reached.

\section{Analysis}

We conducted an inductive thematic analysis using the CFIR as our conceptual framework. ${ }^{28,29}$ Four investigators (CM, MJ, ES, and SK) performed the initial open coding of the data. Investigators met twice during the open coding process to develop and then finalize a codebook of standard definitions for codes. This codebook facilitated coding consistency through the remainder of the analytic process. Two investigators (CM and $\mathrm{MJ}$ ) then independently read and coded all data to ensure intercoder reliability. During this process, $\mathrm{CM}$ and $\mathrm{MJ}$ met ev- ery two weeks to compare coding consistency, resolve discrepancies, and discuss preliminary findings. When the coding was complete, all investigators met to explore and develop themes that encompassed related common codes.

The CFIR was used at two stages of the study: (1) developing the interview guide and (2) cross-checking for any potentially important codes that were missing/needed to be explored further. Thus, the investigators maintained an inductive approach grounded in the data. To assure study rigor, we employed investigator triangulation (use of multiple investigators and participants from multiple clinical roles) and reflexivity (ongoing critique and critical reflection of the individual biases of the investigators). ${ }^{30}$ Coding was performed using Dedoose (version 7.0.23; Los Angeles, California).

\section{RESULTS}

A total of 34 community hospitals completed the PIPA project, of which the project leaders of 25 hospitals connected with the facilitators and were approached to participate; 18 (72\%) hospitals' project leaders participated in the study. We analyzed 18 interviews conducted between facilitators and project leaders, which included a total of 32 project leaders (one to five leaders per interview). The hospitals represented were diverse in geographic location and size (range 4-50 pediatric beds per hospital), and the majority of sites (78\%) supported the trainees (Table 1).

We identified four overarching themes that described the key determinants of pathway implementation in community hospitals. These themes are presented in order of their frequency of occurrence in the data. They included (1) building an implementation infrastructure, (2) engaging and motivating providers, (3) addressing organizational and resource limitations, and (4) devising implementation solutions with facilitators. Descriptions and exemplary quotations for each theme are provided in Table 2 and Appendix Figure 1.

\section{Building an Implementation Infrastructure}

Participants described the importance of building an implementation infrastructure as a critical first step. Establishing an infrastructure required multiple efforts, including forming a team of local champions, delivering didactic education and skills training, and modifying clinical workflows. The multidisciplinary "team of champions" facilitated the division of practical tasks (eg, data entry, Institutional Review Board [IRB] application) and planned educational interventions and setting specific goals, without overloading any given individual. Building an implementation infrastructure "on-the-ground" required thoughtful consideration of local context and engagement of frontline hospital staff commonly involved in the care of children with asthma.

"So, I'm going to sit down with the primary nursing staff and the other four physicians in the group to go over the expectations...We're not going to have the actual EMR [changes] and we're not going to have the nursing documentation field built right away but [we want to] make sure that people are documenting the respiratory score in their generic nursing note so that 
TABLE 1. Demographics of the Participating Community Hospital Sites

\begin{tabular}{|c|c|c|c|c|}
\hline Hospital & Geographic Region & No. of Pediatric Beds & Presence of Trainees & No. of Participants/Site in Interviews \\
\hline B & Midwest & 26 & Yes & 2 \\
\hline C & Midwest & 14 & Yes & 1 \\
\hline D & Southeast & 37 & No & 2 \\
\hline $\mathrm{F}$ & West & 12 & Yes & 1 \\
\hline G & Northeast & 9 & Yes & 1 \\
\hline H & Southwest & 10 & Yes & 2 \\
\hline I & West & 29 & No & 1 \\
\hline L & West & 12 & Yes & 1 \\
\hline M & West & 8 & No & 1 \\
\hline N & West & 32 & Yes & 1 \\
\hline 0 & Midwest & 11 & Yes & 1 \\
\hline$P$ & West & 4 & No & 1 \\
\hline Q & Southwest & 10 & Yes & 2 \\
\hline$R$ & Southeast & 8 & Yes & 3 \\
\hline
\end{tabular}

the information is easily accessible." (Physician leader, Hospital G)

Participants also described the need to deliver education on the evidence supporting changes in practice and skills training specific to pediatric asthma care:

"Once we realized that we were going to be doing this pathway, we started training our nurses on the inpatient side on [pediatric respiratory scoring]." (Nursing leader, Hospital P)

In addition, pathway implementation required modification of clinical workflows via changes to hospital policies or guidelines, electronic medical records (EMR), and/or the physical environment (eg, placing supplies in proximity to care delivery):

"I think it can help if we could get an order set or a nursing protocol where asthmatics over a certain severity can just get steroids in triage." (Physician leader, Hospital A)

\section{Engaging and Motivating Providers}

Another crucial step in pathway implementation was engaging and motivating providers. This included overcoming inertia to practice change, facilitating multidisciplinary collaboration, and handling conflicts regarding practice changes. Participants discussed the excitement of participating in a national collaborative as especially motivating to help drive engage- ment and overcome barriers to change, particularly the ability to compare local hospital performance to national peers.

"I think everyone is a little competitive. So I think that when we see how we compare to other institutionsboth our group and the ER-I think it also adds a little oomph...I think for our nurses too; we're able to say, '[look how we compare to] most of the other hospitals.' I think that helps." (Physician leader, Hospital B)

Multidisciplinary collaboration across a wide variety of frontline pediatric and nonpediatric providers was key to understanding current workflows and identifying needed modifications for pathway implementation:

"I do think clearly our biggest obstacles are the fact that we have adult ED providers. We have the opportunity on the inpatient side [with nursing and respiratory therapy], who really do awesome with pediatric changes, to take our wins where we can and make the changes with the ED. In the ED we have an RN educator. She's very on board with doing the respiratory scoring and getting this whole thing started." (Physician leader, Hospital L)

Intentional communication and leadership skills also played key roles in engaging hesitant providers and handling conflict:

"Just sitting and talking with our respiratory therapist about the ability to provide this type of service or sup- 
TABLE 2. Themes and Example Quotations Regarding the Implementation of an Asthma Protocol in Community Hospitals

\begin{tabular}{lll}
\hline Theme & Sub-theme & Example Quote \\
\hline $\begin{array}{l}\text { Building } \\
\text { Implementation } \\
\text { Infrastructure }\end{array}$ & $\begin{array}{l}\text { Forming team of local } \\
\text { champions }\end{array}$ & $\begin{array}{l}\text { So what I'm going to do over this next two to three weeks, I'm going to sit down with the primary nursing staff and the other four physicians in } \\
\text { the group to go over expectations. I wanted to present the protocol approved through the pediatric safety committee and the medical executive } \\
\text { committee. Hospital G }\end{array}$
\end{tabular}

Delivering didactic We're thinking chest $\mathrm{x}$-rays in the ER, work on that, with some provider education... will probably work the best. He knows that group the best and education so he feels like doing some physician education, like, "this is why we don't do chest $x$-rays. Please don't order them." Then give some evidencebased reasons why we don't. Hospital C

Delivering skills training The lead RT, who was also in this working group for the PIPA project, has reeducated everybody on the use of MDIs and spacers. So from a technical standpoint they know what they are doing now. Hospital A

Modifying current I'm still working with the ED on how we're going to integrate [the asthma evaluation] into triage. Throughput in the ED is such a big deal. Hospital I workflows

\begin{tabular}{|c|c|c|}
\hline \multirow[t]{3}{*}{$\begin{array}{l}\text { Engaging and } \\
\text { Motivating Providers }\end{array}$} & $\begin{array}{l}\text { Overcoming practice } \\
\text { change inertia }\end{array}$ & $\begin{array}{l}\text { I think sometimes it is just like peer pressure... If more and more people do something, then finally that straggler comes along. No one wants to be } \\
\text { seen as the person who's delaying care or prolonging care. Hospital } \mathrm{H}\end{array}$ \\
\hline & $\begin{array}{l}\text { Facilitating multidisciplinary } \\
\text { collaboration and } \\
\text { communication }\end{array}$ & $\begin{array}{l}\text { I think from an ER perspective we'll get there...I think what we're trying to do is develop a long-term relationship and I think that we're ready to } \\
\text { do that....they're invested in trying to provide the best care they can for the kids and to have consistency in care... We have buy in from pharmacy, } \\
\text { respiratory therapy, and nursing. Hospital E }\end{array}$ \\
\hline & Handling conflict & $\begin{array}{l}\text { [Collaborating with the ED] is a little bit difficult...What we really need to do is sit down and look at [the pathway] together and come up with what } \\
\text { makes sense. Because it's just a little awkward, so we have to figure out what works, to be like "what ideas do you have?" Hospital P }\end{array}$ \\
\hline
\end{tabular}

\begin{tabular}{lll}
\hline Addressing & Obtaining support for EMR & $\begin{array}{l}\text { Getting [the respiratory scoring] into Epic would be huge. Honestly as of this moment I'm not sure it is possible because the Epic build will take } \\
\text { time.... We just have to work on updating maybe some Epic templates, our H\&P templates... and maybe creating some smart phrases and things } \\
\text { Resource Limitations }\end{array}$ \\
$\begin{array}{ll}\text { integration } & \text { like that. Hospital A }\end{array}$
\end{tabular}

Resource Limitations

$\begin{array}{ll}\begin{array}{l}\text { Addressing staffing and } \\ \text { capacity shortages }\end{array} & \text { [Training on using respiratory scores] is an added responsibility of the staff and we don't have patient technicians. So nurses are doing everything } \\ \text { from changing the sheets to bringing water to all the medical patients. Hospital B }\end{array}$
capacity shortages from changing the sheets to bringing water to all the medical patients. Hospital B

Navigating institutional We ran into issues with the IRB. I think we might be able to collect January data if in the Board of Supervisor's meeting they're able to approve the review boards IRB. The IRB is submitted, but it's going through [the regional children's hospital]. So it is like an extension because it is a county hospital and then has to be approved by the Board of Supervisors [after the children's hospital]. Hospital F

Working through One of our three goals is to transition to MDI before discharge. The reason there is resistance to [using MDIs] is that in the hospital they were administrative processes having a lot of adult providers with COPD and albuterol PRN. They would be needing a lot of money to dispense all these inhalers that were then never being used...I actually just talked to the direct of pharmacy a couple weeks ago and we do have approval from the CMO of the hospital to go through with this. Hospital N

Devising

Implementation

Solutions with

Practice Facilitators
Figuring out work-arounds Unfortunately, I can't get the protocol through the actual EMR....we're not going to have the nursing documentation field built right away but make sure that people are documenting the respiratory score just in their generic nursing note so that the information is easily accessible. What want the nurses to do is to be assessing the patient prior to every treatment and deciding whether or not this child would need to wean. Hospital $\mathrm{G}$

Proactive coaching Facilitator: Because it might [help to] have a nurse champion that maybe if it came from their end to push for some of these things...might help to be a motivating factor for the institution as a whole. Project leader: I don't know if we have anybody that high up enough.

Facilitator: I don't necessarily know from a nursing end if you need anyone that high. You just need someone enthusiastic that's willing to ask people in leadership questions and say, "We need this. I already have someone who can do it. But I need the hospital to support it." Project leader: Yeah. I think that's right. Yeah. We can definitely try. Hospital B

Just-in-time solution building

Facilitator: Basically, everyone has sort of said that it's almost impossible for [EMR changes] to happen and it would probably take eight to nine months for it to get approved through Epic. We're thinking about doing modifying the existing asthma order set and using that as individuals. I can create it, and everyone could just adapt the order set that I created.

Project leader: At another community site, we just [placed an order that read], "Follow the Asthma Pathway." Then, the nurse or respiratory therapist... would go pick up their Asthma Pathway and follow that. Lots of ways to do it. Hospital P

Abbreviations: $\mathrm{CMO}$, chief medical officer; COPD, chronic obstructive pulmonary disease; ED, emergency department; EMR, electronic medical record; ER, emergency room; H\&P, history and physical; IRB, institutional review board; MDI, metered dose inhaler; PRN, pro re nata ("as needed"); RT, respiratory therapy; X-ray, radiograph

port and seeing what their reservations have been, at least it's open to conversation so that we could provide these types of therapies in the future and we're able to see like what people's concerns are. I think just basically increasing familiarity with not only these processes, but different types of therapy will hopefully in the future help us provide better care to our patients." (Physician leader, Hospital Q)
Addressing Organizational and Resource Limitations Participants recognized organizational and resource limitations, some of which may be unique to community hospitals that prioritize resources for adult care. The limitations described included EMR staff support, healthcare provider staffing/capacity, navigating IRBs, and addressing administrative processes. Competing demands for information technology staff support and lack of prioritization of pediatric-specific ini- 
tiatives often hindered efforts to modify the EMR.

"Resource wise, we are hoping to implement an order set in our Epic EMR, [but] finding the availability from the Epic team may be a challenge." (Physician Leader, Hospital A)

Participants also reported that limited staff capacity (eg, nursing, RT) hindered pathway implementation efforts. This limited capacity hindered workflow changes and limited the time available for education and training on pathways:

"[Respiratory scoring for asthma is] an added responsibility for the [nursing] staff and we don't have patient technicians. So they're doing everything from changing the sheets to bringing water to all of the medical patients. So, that I think may be a barrier." (Physician leader, Hospital B)

Across sites, navigating the IRB posed various challenges. Some sites were required to obtain approval from regional IRBs, which did not have resources to devote to pediatric projects. Other sites did not have IRBs at all, but instead required separate approvals for the project from hospital leadership or other entities:

"On the IRB, I contacted the manager of the IRB and she's said, 'No, it's not an IRB project,' but she sent it to another director for review, and it took forever to be able to get a data agreement with [the local university hospital] so that we can pull the data. I just couldn't believe it took months to get done." (Physician Leader, Hospital K)

Finally, administrative barriers such as addressing formulary changes in the context of adult-focused settings were challenging. For example, at one hospital, metered dose inhalers (MDIs) were not used for adult patients, and the hospital administration was resistant to incorporate their use into practice for pediatric patients due to the cost of such changes.

"The [general hospital] didn't have MDI's anymore because of cost reasons, and when we started the pediatric work, we really made it a point to get the MDI's for pediatric patients back in the formulary." (Physician leader, Hospital A)

\section{Devising Implementation Solutions with Practice Facilitators}

Participants often devised pathway implementation solutions with facilitators in-the-moment during meetings. This problem-solving included figuring out work-arounds, proactive coaching by external facilitators, and just-in-time solution building. Furthermore, in meetings that included more than one project leader, leaders would often work with each other to devise solutions. Meetings provided forums that stimulated identification of implementation barriers, brainstorming, and subsequently solution building.

Physician leader: I'm wondering if we could, as an interim solution, try out an algorithm on paper, I don't know if that's allowed, until we get Epic approval. Do you know?

Nurse Leader: You mean having an algorithm posted in triage? Yeah, I don't see why not. (Hospital A)
Next, problem solving was often driven by the facilitator's experience and knowledge, drawn from their interactions with other collaborative sites or their own prior experiences with asthma, Ql, or pathway implementation. The facilitators brought an outside perspective, not bound by that particular hospital's local culture or structural intricacies. This proactive coaching spurred the identification of creative, yet practical solutions:

Project Leader: We're still trying to get all our templates [for the EMR]... because [currently they are] all adult templates.

Facilitator: If you're making templates right now, could you also add the three asterisks? Like smoking or exposure to second hand tobacco smoke or marijuana... then have the three asterisks there and then "Referral made? ${ }^{\star * *}$. That would force people to document in a certain place in the template as well.

Project Leader: That's definitely something we could add right now. (Hospital $O$ )

Check-in meetings with facilitators offered an opportunity to trouble shoot, brainstorm work-arounds, devise in-the-moment site-specific solutions to enable successful pathway implementation, and provide ongoing support throughout implementation.

\section{DISCUSSION}

Pathways can improve the quality of care for children with asthma. ${ }^{31}$ However, there is little evidence-based guidance on how to implement pathways and improve pediatric care in community hospitals, ${ }^{17-20}$ where the majority of children are cared for nationally. This is the first study to our knowledge that details the key determinants of pediatric asthma pathway implementation in community hospital settings. We identified four key determinants of implementation that can help guide others in similar settings. These include building an implementation infrastructure, engaging and motivating multidisciplinary providers, addressing organizational and resource limitations, and using external facilitators to devise implementation solutions.

Existing frameworks such as the CFIR outline the potential determinants of implementation success but do not provide population- or setting-specific guidance. ${ }^{27}$ There have been prior studies detailing pathway implementation for pediatric populations, but these studies did not focus on community hospitals. ${ }^{32,33}$ Our findings align with these prior studies, which highlight the importance of identifying implementation champions, engaging and motivating multidisciplinary providers, establishing a $\mathrm{Ol}$ infrastructure, and addressing organizational and resource limitations, such as EMR integration. ${ }^{32,33}$ However, our study provides unique insights into issues that are important to successful pathway implementation in community hospitals, including engagement of adult-focused healthcare providers, reprioritization of resources toward the care of children, and the potentially critical role of external facilitators.

Our findings indicate that community hospitals seeking to improve care for children may particularly benefit from using external facilitators and/or partnering with external organizations. 
We found that external facilitators played a significant and proactive role in community hospitals' efforts to improve care for children. Facilitators helped devise work-arounds and engaged in just-in-time solution building with local project leaders. For instance, facilitators helped develop strategies for training healthcare providers in performing new clinical tasks, building reminders of pathway recommendations into clinical workflows, and overcoming resource barriers. Thus, community hospitals may uniquely benefit from participation in national learning collaboratives, which often provide avenues for external facilitation. ${ }^{25,34,35}$ National networks, such as the VIP network, lead national learning collaboratives that provide external facilitation as well as other resources (eg, educational materials, data analysis support) to community hospitals seeking to improve pediatric care. ${ }^{24}$ Previous work by McDaniel et al. identified that intentional partnerships between children's and community hospitals can also potentially provide access to resources for education and training in pediatric care and support in navigating organizational and resource challenges. ${ }^{22}$

Our results characterize the key determinants of pediatric asthma pathway implementation using a national sample of community hospitals that were diverse in geography, size, and structure. This imparts greater transferability of our findings. We also used strategies to promote the rigor of our findings, including triangulation and reflexivity. However, our study has several limitations. First, we analyzed only the meetings that occurred during the early months of pathway implementation. As such, we did not capture any key determinants that may have arisen later in implementation. However, process analyses of implementation indicate that the majority of implementation efforts occurred within these first three to four months. ${ }^{36}$ Second, we did not elicit input from hospital administration or leadership. The lack of administrative/leadership input probably affected the CFIR themes we found, as no themes from the outer setting were elicited. However, the goal of our study was to characterize the experiences of those leading implementation efforts, and focusing on these leaders allows our work to better guide those doing similar work in the future. Third, we used CFIR to guide the development of our interview guide and as a reference during analysis, which may have skewed our findings to preferentially reflect CFIR constructs. However, our overall analysis was grounded in the primary data and we employed reflexivity during all stages of our analysis. In addition, having the facilitators conduct the qualitative interviews may have biased our findings toward the perspectives of the facilitators; however, the facilitators represented quite diverse clinical and $\mathrm{Ql}$ backgrounds. Finally, our findings do not necessarily correlate with improvements in clinical outcomes. As such, they are not meant to serve as explicit recommendations for improving patient outcomes, but rather as a characterization of the context, processes, and experiences of implementing pathways in the community setting to inform others doing this important work.

\section{CONCLUSIONS}

We identified the key determinants of pediatric asthma pathway implementation in community hospitals, which may help inform Ql efforts in these settings. We also identified organizational and resource limitations that are probably unique to these adult-focused hospitals. Participating in national learning collaboratives and/or working with facilitators may support pathway implementation and improved quality of care for children with asthma in community hospitals.

Future work should seek to correlate these and other determinants of pathway implementation with health outcomes for hospitalized children, as well as integrate broader and more diverse samples of community hospitals.

Disclosures: The authors have no conflicts of interest or corporate sponsors for this manuscript. Each author participated in the development of this manuscript, including the development and implementation of methods, analysis of data, and preparation of the manuscript. All authors have reviewed the submitted manuscript and approved the manuscript for submission.

Funding: This project was supported through the Value in Inpatient Pediatrics Network. The funding source was not involved in study design, data collection, analysis, writing of this manuscript, or decision to submit for publication.

\section{References}

1. National Asthma E, Prevention P. Expert Panel Report 3 (EPR-3): Guidelines for the diagnosis and management of asthma-summary report 2007. J Allergy Clin Immunol. 2007;120(5):S94-S138. https://doi.org/10.1016/j. jaci.2007.09.043

2. Bekmezian A, Hersh AL, Maselli JH, Cabana MD. Pediatric emergency departments are more likely than general emergency departments to treat asthma exacerbation with systemic corticosteroids. J Asthma. 2011;48(1):6974. https://doi.org/10.3109/02770903.2010.535884.

3. Biagini Myers JM, Simmons JM, Kercsmar CM, et al. Heterogeneity in asthma care in a statewide collaborative: the Ohio Pediatric Asthma Repository. Pediatrics. 2015;135(2):271-279. https://doi.org/10.1542/peds.2014-2230.

4. Kharbanda $A B$, Hall M, Shah SS, et al. Variation in resource utilization across a national sample of pediatric emergency departments. J Pediatr. 2013;163(1):230-236. https://doi.org/10.1016/j.jpeds.2012.12.013.

5. O'Connor MG, Saville BR, Hartert TV, Arnold DH. Treatment variability of asthma exacerbations in a pediatric emergency department using a severity-based management protocol. Clin Pediatr (Phila). 2014;53(13):1288-1290. https://doi.org/10.1177/0009922813520071.

6. Lougheed MD, Garvey N, Chapman KR, et al. Variations and gaps in management of acute asthma in Ontario emergency departments. Chest. 2009;135(3):724-736. https://doi.org/10.1378/chest.08-0371.

7. Bekmezian A, Fee C, Weber E. Clinical pathway improves pediatrics asthma management in the emergency department and reduces admissions. J Asthma. 2015;52(8):806-814. https://doi.org/10.3109/02770903.2015.1019086.

8. Chen KH, Chen CC, Liu HE, Tzeng PC, Glasziou PP. Effectiveness of paediatric asthma clinical pathways: a narrative systematic review. J Asthma. 2014;51(5):480-492. https://doi.org/10.3109/02770903.2014.887728.

9. Johnson KB, Blaisdell CJ, Walker A, Eggleston P. Effectiveness of a clinical pathway for inpatient asthma management. Pediatrics. 2000;106(5):10061012. https://doi.org/10.1542/peds.106.5.1006.

10. Kelly CS, Andersen CL, Pestian JP, et al. Improved outcomes for hospitalized asthmatic children using a clinical pathway. Ann Allergy Asthma Immunol. 2000;84(5):509-516. https://doi.org/10.1016/S1081-1206(10)62514-8.

11. McDowell KM, Chatburn RL, Myers TR, O'Riordan MA, Kercsmar CM. A cost-saving algorithm for children hospitalized for status asthmaticus. Arch Pediatr Adolesc Med. 1998;152(10):977-984. https://doi.org/10.1001/archpedi.152.10.977.

12. Miller AG, Breslin ME, Pineda LC, Fox JW. An asthma protocol improved adherence to evidence-based guidelines for pediatric subjects with status asthmaticus in the emergency department. Respir Care. 2015;60(12):17591764. https://doi.org/10.4187/respcare.04011.

13. Nkoy F, Fassl B, Stone B, et al. Improving pediatric asthma care and outcomes across multiple hospitals. Pediatrics. 2015;136(6):e1602-e1610. https://doi.org/10.1542/peds.2015-0285.

14. Rutman L, Atkins RC, Migita R, et al. Modification of an established pediatric asthma pathway improves evidence-based, efficient care. Pediatrics. 
2016;138(6). https://doi.org/10.1542/peds.2016-1248.

15. Glauber JH, Farber HJ, Homer CJ. Asthma clinical pathways: toward what end? Pediatrics. 2001;107(3):590-592. https://doi.org/10.1542/ peds.107.3.590.

16. Grimshaw J, Eccles M, Thomas R, et al. Toward evidence-based quality improvement. Evidence (and its limitations) of the effectiveness of guideline dissemination and implementation strategies 1966-1998. J Gen Intern Med. 2006;21(2):S14-S20. https://doi.org/10.1111/j.1525-1497.2006.00357.x.

17. Scott SD, Grimshaw J, Klassen TP, Nettel-Aguirre A, Johnson DW. Understanding implementation processes of clinical pathways and clinical practice guidelines in pediatric contexts: a study protocol. Implement Sci. 2011;6:133. https://doi.org/10.1186/1748-5908-6-133.

18. Walls TA, Hughes NT, Mullan PC, Chamberlain JM, Brown K. Improving pediatric asthma outcomes in a community emergency department. Pediatrics. 2017;139(1). https://doi.org/10.1542/peds.2016-0088.

19. Kaiser SV, Lam R, Cabana MD, et al. Best practices in implementing inpatient pediatric asthma pathways: a qualitative study. J Asthma. 2019:1-11. https:// doi.org/10.1080/02770903.2019.1606237

20. Leyenaar JK, Ralston SL, Shieh MS, Pekow PS, Mangione-Smith R, Lindenauer PK. Epidemiology of pediatric hospitalizations at general hospitals and freestanding children's hospitals in the United States. J Hosp Med. 2016;11(11):743-749. https://doi.org/10.1002/jhm.2624.

21. Franca UL, McManus ML. Availability of definitive hospital care for children JAMA Pediatr. 2017;171(9):e171096. https://doi.org/10.1001/jamapediatrics.2017.1096

22. McDaniel CE, Jennings $R$, Schroeder AR, Paciorkowski N, Hofmann M, Leyenaar J. Aligning inpatient pediatric research with settings of care: a call to action. Pediatrics. 2019;143(5). https://doi.org/10.1542/peds.2018-2648.

23. Kaiser SV JB. Value in inpatient pediatrics network launches National Asthma Project. In: AAP Quality Connections 2018; 26:8-9. Retrieved from: https:// www.aap.org/en-us/Documents/coqips_newsletter_2018_winter_26.pdf

24. Value in Inpatient Pediatrics. https://www.aap.org/en-us/professional-resources/quality-improvement/Pages/Value-in-Inpatient-Pediatrics.aspx. Accessed December 1, 2017

25. The Breakthrough Series: IHI's Collaborative Model for Achieving Breakthrough Improvement. IHI Innovation Series white paper. Boston: Institute for Healthcare Improvement; 2003. Retrieved from: www.IHI.org

26. Powell BJ, Waltz TJ, Chinman MJ, et al. A refined compilation of implementa- tion strategies: results from the Expert Recommendations for Implementing Change (ERIC) project. Implement Sci. 2015;10:21. https://doi.org/10.1186/ s13012-015-0209-1.

27. Damschroder LJ, Aron DC, Keith RE, Kirsh SR, Alexander JA, Lowery JC. Fostering implementation of health services research findings into practice: a consolidated framework for advancing implementation science. Implement Sci. 2009;4:50. https://doi.org/10.1186/1748-5908-4-50

28. Braun VaC, V. Thematic analysis. In: H. Cooper PC, Long DL, Panter AT, Rindskopf E, Sher KJ, eds. APA handbook of research methods in psychology, Vol 2. Research designs: Quantitative, qualitative, neuropsychologial, and biological. Washington, DC, US: American Psychological Association; 2012. https://doi.org/10.1037/13620-000.

29. Charmaz K. Grounded Theory. 2nd ed. Thousand Oaks, CA: SAGE Publications; 2014.

30. Creswell JW, Poth CNCN CJaP. Qualitative Inquiry and Research Design: Choosing Among Five Approaches. Thousand Oaks, CA: Sage; 2017.

31. Kaiser SV, Rodean J, Bekmezian A, et al. Effectiveness of pediatric asthma pathways for hospitalized children: a multicenter, national analysis. J. Pediatr. 2018;197:165-171. https://doi.org/10.1016/j.jpeds.2018.01.084.

32. Leyenaar JK, Andrews CB, Tyksinski ER, Biondi E, Parikh K, Ralston S. Facilitators of interdepartmental quality improvement: a mixed-methods analysis of a collaborative to improve pediatric community-acquired pneumonia management. BMJ Qual Saf. 2019;28(3):215-222. https://doi.org/10.1136/ bmjqs-2018-008065.

33. Ralston SL, Atwood EC, Garber MD, Holmes AV. What works to reduce unnecessary care for bronchiolitis? A qualitative analysis of a national collaborative. Acad Pediatr. 2017;17(2):198-204. https://doi.org/10.1016/j. acap.2016.07.001

34. Parikh K, Biondi E, Nazif J, et al. A multicenter collaborative to improve care of community acquired pneumonia in hospitalized children. Pediatrics. 2017;139(2). https://doi.org/10.1542/peds.2016-1411.

35. Ralston S, Garber M, Narang S, et al. Decreasing unnecessary utilization in acute bronchiolitis care: results from the value in inpatient pediatrics network. J Hosp Med. 2013;8(1):25-30. https://doi.org/10.1002/jhm.1982.

36. Gupta N CA, Cabana MD, Jennings B, Parikh K, Kaiser SV. PIPA (Pathways for Improving Pediatric Asthma Care): Process Evaluation of a National Collaborative to Implement Pathways. Platform presented at Pediatric Academic Society National Meeting. Baltimore, Maryland; 2019. 